\title{
Estudo comparativo de eco-Doppler com arteriografia na avaliação da doença oclusiva aorto-ilíaca
}

\author{
Comparative study of Doppler ultrasonography with arteriography in \\ the evaluation of aortoiliac occlusive disease
}

\author{
Ricardo Cesar Rocha Moreira*
}

\section{Resumo}

Contexto: A arteriografia com contraste (AC) tem sido o exame tradicional de avaliação de pacientes com suspeita de doença oclusiva aorto-ilíaca (DOAI). Recentemente, métodos menos invasivos, como a eco-Doppler, têm sido usados com a mesma finalidade.

Objetivo: Comparar prospectivamente a eco-Doppler com a AC e eventual manometria arterial direta (MAD) na avaliação pré-operatória de pacientes com suspeita de DOAI.

Métodos: Foram submetidos a eco-Doppler e a AC 125 pacientes internados para tratamento de doença arterial oclusiva dos membros inferiores, avaliando comparativamente 552 segmentos da aorta infrarrenal e das artérias ilíacas comum e externa. As lesões encontradas foram classificadas em cinco categorias: 1) normal e estenose leve ( 0 a 19\%); 2) estenose moderada (20 a 49\%); 3) estenose significativa (50 a $79 \%)$; 4) estenose crítica (80 a 99\%); e 5) oclusão total. A MAD foi usada em 19 segmentos de 15 pacientes para classificar lesões limítrofes entre duas categorias. Foram calculados índices de validade (sensibilidade, especificidade, valor preditivo positivo, valor preditivo negativo e acurácia) para distinguir lesões hemodinamicamente significativas de não-significativas e para distinguir estenoses críticas de oclusões. O padrão-ouro foi AC, complementado pela MAD. Foram também calculados coeficientes de correlação kappa entre arteriografias e eco-Doppler para o conjunto dos segmentos aorto-ilíacos.

Resultados: Lesões clinicamente relevantes (estenoses de 50 a $99 \%$ e oclusões totais) foram observadas na eco-Doppler em 163 segmentos $(29,5 \%)$ e na AC em 158 segmentos (28,6\%). A eco-Doppler mostrou altos índices de validade para distinguir lesões hemodinamicamente significativas de lesões não-significativas em todos os segmentos (acurácia $=92 \% ;$ kappa $=0,81$ ) e para diferenciar estenoses críticas de oclusões (acurácia $=86 \%$; kappa $=0,73$ ). Os índices de correlação entre os resultados das eco-Doppler e das AC foram ótimos em todos os segmentos aorto-ilíacos.

Conclusão: A eco-Doppler apresenta elevados índices de validade e ótimos coeficientes de correlação com a AC na avaliação de pacientes com suspeita de DOAI.

Palavras-chave: Aorta abdominal, artéria ilíaca, aterosclerose, angiografia, eco-Doppler.

\begin{abstract}
Background: Contrast arteriography (CA) has been the traditional method of evaluation of patients with suspected aortoiliac occlusive disease (AIOD). Recently, less invasive methods, such as Doppler ultrasonography, have been used for the same purpose.
\end{abstract}

Objective: The present study prospectively compares Doppler ultrasonography with CA and direct arterial manometry (DAM) in the preoperative evaluation of patients with suspected AIOD.

Methods: A total of 125 patients admitted for treatment of arterial occlusive disease of the lower extremities underwent Doppler ultrasonography and CA, comparatively evaluating 552 aortic, common iliac e external iliac segments. The lesions found were classified into five categories: 1) normal and mild stenosis $(0-19 \%) ; 2)$ moderate stenosis (20-49\%); 3) significant stenosis (50-79\%); 4) critical stenosis (80-99\%); and 5) total occlusion. DAM was used in 19 segments of 15 patients to classify borderline lesions. Validity indexes (sensitivity, specificity, positive predictive value, negative predictive value and overall accuracy) were calculated to distinguish hemodynamically significant from non-significant stenosis and to discriminate critical stenosis from total occlusions. The gold standard was CA, supplemented by DAM. Correlation coefficients (kappa statistics) between arteriography and Doppler ultrasonography were also calculated for the whole sample of aortoiliac segments.

Results: Clinically relevant lesions (stenoses between $50-99 \%$ and total occlusions) were observed on Doppler ultrasonography in 163 segments $(29.5 \%)$ and on CA in 158 segments $(28.6 \%)$. Doppler ultrasonography showed high validity indexes to distinguish hemodynamically significant from non-significant lesions (overall accuracy $=92 \% ; \mathrm{kappa}=0.81)$ and an overall accuracy of $86 \%(\mathrm{kappa}$ $=0.73$ ) to distinguish critical stenosis from total occlusion. Optimal correlation coefficients between the results of Doppler ultrasonography and CA were observed for all aortoiliac segments.

Conclusion: Doppler ultrasonography has high validity indexes and optimal correlation coefficients with CA in the evaluation of patients with suspected AIOD.

Keywords: Abdominal aorta, iliac artery, atherosclerosis, angiography, Doppler ultrasonography.

* Serviço de Cirurgia Vascular, Hospital Universitário Cajuru (HUC), Pontifícia Universidade Católica do Paraná (PUC-PR), Curitiba PR.

Não foram declarados conflitos de interesse associados à publicação deste artigo.

Artigo submetido em 19.11.07, aceito em 12.12.08.

J Vasc Bras. 2009;8(1):3-13.

Copyright $\odot 2009$ by Sociedade Brasileira de Angiologia e de Cirurgia Vascular 


\section{Introdução}

A arteriografia com contraste iodado (AC) é o exame tradicional para o diagnóstico da doença oclusiva aortoilíaca (DOAI) ${ }^{1}$. A AC mostra claramente as alterações morfológicas provocadas pela aterosclerose na aorta abdominal e nas artérias ilíacas, permitindo planejar o tratamento. Apesar de consagrada pela tradição, a AC apresenta sérias desvantagens como método diagnóstico: é invasiva, desconfortável, traz o risco de complicações graves e tem custo elevado ${ }^{2}$. Nas últimas 2 décadas, a eco-Doppler, exame que reúne, num só aparelho, a imagem do vaso sanguíneo pela ultrassonografia, e a análise do fluxo sanguíneo pelo efeito Doppler, vem sendo usada na avaliação inicial da doença arterial oclusiva dos membros inferiores ${ }^{3,4}$. A eco-Doppler apresenta as seguintes vantagens em relação à AC: não é invasiva; fornece informações anatômicas e fisiológicas; é muito mais barata e o risco de complicações é nulo ${ }^{5}$.

A maior desvantagem da eco-Doppler é depender da habilidade e experiência do examinador ${ }^{6}$. Por esse motivo, a eco-Doppler ainda é menos usada na avaliação dos vasos do tronco do que dos vasos das extremidades e do pescoço. Apesar de amplamente difundida, persistem dúvidas quanto à acurácia da eco-Doppler nos pacientes com múltiplas lesões ateroscleróticas ${ }^{7,8}$. Em particular, a tomada de decisões clínicas baseada nos dados ultrassonográficos ainda é objeto de controvérsias 9 .

O objetivo do presente trabalho foi determinar os índices de validade da eco-Doppler e sua correlação com a AC na avaliação de pacientes com suspeita de DOAI.

\section{Métodos}

Pacientes internados no Serviço de cirurgia Vascular do Hospital Nossa Senhora das Graças e na Disciplina de Cirurgia Vascular do Hospital de Clínicas da Universidade Federal do Paraná (UFPR), ambos em Curitiba (PR), foram recrutados para participar do estudo, de forma prospectiva. Todos os pacientes haviam sido internados com diagnóstico de isquemia de membros inferiores, para possível tratamento cirúrgico ou intervencionista. O exame clínico inicial consistiu, na história clínica, em exame físico e avaliação das artérias dos membros inferiores com fluxômetro Doppler portátil.

No total, foram estudados prospectivamente 125 pacientes nos quais, após a avaliação clínica inicial, existia a suspeita de DOAI ou de doença oclusiva em múltiplos níveis. Foram 99 homens $(79,2 \%)$ e 26 mulheres $(20,8 \%)$, com média de idade de $62 \pm 8$ anos. As doenças associadas estão descritas na Tabela 1 .

Com base no exame inicial, o paciente foi colocado numa das categorias funcionais de doença arterial oclusiva dos membros inferiores, conforme os padrões recomendados pela Joint Commission on Reporting Standards da Society for Vascular Surgery e da North American Chapter/International Society for Cardiovascular Surgery ${ }^{10}$.

Neste estudo, a aorta abdominal e as artérias ilíacas foram divididas em três segmentos: aorta infrarrenal (da origem das artérias renais à bifurcação); artéria ilíaca comum (da origem da aorta à bifurcação) e artéria ilíaca externa (da bifurcação da ilíaca comum até a transição para a artéria femoral, no ligamento inguinal).

Os exames de eco-Doppler foram realizados por dois ecografistas vasculares titulados. Todos os exames foram realizados de acordo com o protocolo do exame de eco-Doppler.

\section{Protocolo do exame de eco-Doppler}

Preparo do paciente:

- Jejum de 12 horas (exames agendados para o período matinal);

- Bexiga vazia de urina, ou pelo menos o esvaziamento máximo possível, logo antes de iniciar o exame;

- Antifiséticos não são usados de forma rotineira (eventualmente, quando a quantidade de gases intestinais impede um exame tecnicamente adequado, são empregados laxante e simeticone por 1 a 3 dias e repetido o exame);

- Repouso de pelo menos 30 minutos antes do exame. Técnica do exame:

- Pacientes em decúbito dorsal, em maca apropriada;

- Membros inferiores em extensão para o exame do segmento aorto-ilíaco (ressalva: para o adequado exame 
Tabela 1 - Doenças associadas

\begin{tabular}{lcc}
\hline Doença & $\mathbf{N}^{\mathbf{0}}$ de pacientes & $\%$ \\
\hline Tabagismo & 88 & 70,4 \\
Hipertensão arterial & 65 & 52,0 \\
Doença coronariana & 45 & 36,0 \\
Diabetes melito & 36 & 28,8 \\
Doença pulmonar obstrutiva crônica & 29 & 23,2 \\
Insuficiência cardíaca & 13 & 10,4 \\
Doença cerebrovascular & 11 & 8,8 \\
Outras* & 24 & 19,2 \\
\hline
\end{tabular}

* Dez ou menos casos de: obesidade (8); dislipidemia (5); insuficiência renal crônica (4); etilismo (5); isquemia mesentérica (1); câncer de coxa (1).

do segmento aorto-ilíaco às vezes é necessária a adoção de decúbitos laterais);

- Abdução e rotação externa do membro para exame das femorais varreduras transversais, longitudinais e oblíquas, obtendo as imagens e os registros que interessam.

Imagem modo B (preto e branco, imagem da ecografia convencional): permite medir calibres, determinar dilatações, identificar trombos, placas, etc.

Imagem colorida (incluindo o uso do modo angio ou power-Doppler): permite determinar a presença ou ausência de fluxo, localizar áreas de estenose (por características como mudança no mosaico de cores, turbulência, afinamento na seç̧ão colorida quando se usa o modo angio, etc.).

Registro Doppler fluxométrico: permite as medidas precisas de velocidades (sistólica de pico ou máxima, diastólica final e velocidade média) e a obtenção de índices ou tempos, como: índices de resistência, pulsatilidade, aceleração, tempo de aceleração, etc.

Teste de hiperemia reativa: colocação de manguito na coxa do paciente, insuflado até $50 \mathrm{mmHg}$ acima da pressão sistólica. O manguito é mantido insuflado por 3 a 5 minutos e desinsuflado rapidamente, com novo registro do fluxo com análise da forma de onda na artéria femoral após 30 segundos.

Os critérios ultrassonográficos para classificar as lesões são apresentados no Anexo.

As lesões detectadas foram classificadas em cinco categorias, de acordo com os parâmetros observados e medidos na eco-Doppler a cores (Tabela 2).

Todos os pacientes foram submetidos a AC, de acordo com o protocolo específico do estudo protocolo

Tabela 2 - Classificação das lesões quanto ao grau de estenose

\begin{tabular}{lcc}
\hline Grau & Categoria & Redução do diâmetro \\
\hline 1 & Normal ou estenose leve & $0-19 \%$ \\
2 & Moderada (não-significativa) & $20-49 \%$ \\
3 & Significativa & $50-79 \%$ \\
4 & Grave ou "crítica" & $80-99 \%$ \\
5 & Oclusão & $100 \%$ \\
\hline
\end{tabular}


do exame arteriográfico, que incluía pelo menos duas injeções de contraste e filmagem em planos diferentes.

\section{Protocolo do exame arteriográfico}

Preparo do paciente:

- Jejum de 4 a 6 horas;

- Exames agendados de preferência para o período matinal;

- Tricotomia das regiões inguinais (e eventualmente da região axilar direita).

Técnica do exame:

- Pacientes em decúbito dorsal (e depois oblíquo) na mesa de exame radiográfico;

- Membros inferiores em extensão;

- Preparo da região anatômica a ser puncionada com tintura ou solução tópica de iodo-pirrolidona a $2 \%$ e colocação de campos estéreis;

- Local da punção: artéria femoral direita. Se o pulso femoral direito não é palpável: punção da femoral esquerda. Se ambos os pulsos femorais não são palpáveis: punção da artéria axilar direita;

- Punção com agulha e mandril, 20 G;

- Obtenção de fluxo pulsátil pela agulha e introdução de fio-guia 0,035 ;

- Passagem do fio-guia até a aorta abdominal, sob controle fluoroscópico;

- Introdução do cateter "multipurpose" 5 ou 7F com 140 cm de comprimento;

- Colocação do cateter com a ponta ao nível das artérias renais;

- Conexão do cateter à bomba injetora;

- Injeção de contraste iodado (aproximadamente 60 $\mathrm{mL}$ );

- Obtenção das imagens do segmento aorto-ilíaco em projeções ântero-posterior e oblíqua anterior direita ou esquerda;

- Nova injeção do contraste iodado para as imagens do membro inferior, a partir das artérias femorais. Se as imagens forem satisfatórias, retirada do cateter;
- Compressão manual do local da punção por 10 minutos, no mínimo;

- Se persistem dúvidas quanto ao significado hemodinâmico de uma estenose, procede-se à manometria arterial direta (MAD).

Todas as AC foram realizadas em equipamentos radiológicos com imagem de subtração digital. Nos exames em que persistiram dúvidas quanto ao significado hemodinâmico de alguma lesão, foi realizada a MAD.

\section{Protocolo da MAD}

Casos de estenose ipsilateral ao cateter:

- Arteriografia com cateter em pelo menos dois planos (AP e uma projeção oblíqua);

- Conexão do cateter a um transdutor de pressão de membrana, ligado a um monitor eletrônico que fornece curva de pressão em tempo real;

- MAD na aorta abdominal, proximalmente a qualquer lesão;

- Medida do gradiente através da lesão estenótica, pela retirada lenta do cateter e observação das pressões ao longo da artéria;

- Injeção de papaverina $(30 \mathrm{mg})$ distalmente à lesão estenótica e nova MAD 2 a 3 minutos depois.

Casos de estenose contralateral ao cateter:

- Punção da artéria femoral contralateral com agulha ou com cateter curto tipo Jelco $18 \mathrm{~F}$.

- MAD na aorta abdominal através do cateter (como descrito acima);

- Conexão da agulha/cateter curto ao transdutor;

- MAD contralateral em repouso;

- Medida da PAD depois da injeção de papaverina (30 $\mathrm{mg}$ ) através da agulha ou do cateter curto colocado na luz da femoral contralateral.

Um gradiente maior que $15 \mathrm{mmHg}$ entre o segmento proximal e distal a uma estenose foi considerado hemodinamicamente significativo. Os exames foram interpretados pelo radiologista vascular, que forneceu o laudo, com base nas imagens e na MAD.

As lesões encontradas foram classificadas nas mesmas cinco categorias do exame de eco-Doppler, de 
Tabela 3 - Classificação funcional dos pacientes de acordo com o grau de isquemia $(n=125)$

\begin{tabular}{lccc}
\hline Grau de isquemia & $\begin{array}{c}\text { Apresentação clínica dos } \\
\text { pacientes }\end{array}$ & No de pacientes & $\mathbf{( \% )}$ \\
\hline 1 & Claudicação leve & $3^{*}$ & 2,4 \\
2 & Claudicação moderada & $8^{*}$ & 6,4 \\
3 & Claudicação incapacitante & 13 & 10,4 \\
4 & Dor em repouso & 33 & 26,4 \\
5 & Lesão trófica limitada & 67 & 53,6 \\
\hline
\end{tabular}

* Os 11 pacientes nas categorias 1 e 2 tinham aneurismas aorto-ilíacos.

acordo com o grau de estenose. As lesões classificadas nas categorias estenose significativa (grau 3), crítica (grau 4) e oclusão (grau 5) foram consideradas clinicamente relevantes. No presente trabalho, clinicamente relevante significa que a lesão era a causa da isquemia ou provocava redução do influxo para o membro, podendo comprometer os resultados de reconstrução arterial distal à lesão.

Foram excluídos da análise comparativa:

- Os segmentos ilíacos proximais a um membro inferior amputado, por serem clinicamente irrelevantes;

- Os segmentos ilíacos contralaterais aparentemente normais em pacientes com isquemia unilateral. Por razões éticas, não se justificavam injeções adicionais de contraste, nem MAD nesses segmentos. Portanto, não foi possível estabelecer em tais segmentos um padrão-ouro que permitisse a comparação com a ecoDoppler;

- Segmentos ilíacos onde não foi possível realizar exame ultrassonográfico satisfatório, por razões técnicas (calcificação arterial extensa, meteorismo intestinal).

Em 95 pacientes, o eco-Doppler foi realizado antes da $\mathrm{AC}$; nos demais 30 pacientes, a ordem dos exames foi inversa. $\mathrm{O}$ intervalo médio entre os dois exames foi 2,5 dias (no máximo, 15 dias). O laudo ultrassonográfico só foi colocado no prontuário do paciente depois de realizado o exame arteriográfico e vice-versa. Dessa forma, tanto os ultrassonografistas quanto os radiologistas vasculares desconheciam o resultado do exame comparativo.
Os pacientes foram informados da natureza observacional do estudo, e que o exame de ultrassonografia não traria qualquer risco para sua saúde. Autorização por escrito foi obtida de todos os pacientes para realização dos exames arteriográficos. O protocolo do estudo foi aprovado pelas Comissões de Ética em Pesquisa do Hospital Nossa Senhora das Graças e do Hospital de Clínicas da UFPR.

Os resultados foram analisados estatisticamente através de dois métodos: índices de validade e coeficiente de correlação kappa. Os índices de validade: sensibilidade, especificidade, valor preditivo positivo e valor preditivo negativo e acurácia foram calculados para cada um dos segmentos e para toda a amostra. Os coeficientes de correlação kappa entre os resultados das AC e eco-Doppler foram calculados para cada segmento. Esse índice permite a correção da influência do acaso sobre os resultados da correlação entre os dois exames.

\section{Resultados}

Os 125 pacientes foram classificados em categorias funcionais de doença arterial oclusiva dos membros inferiores, como descrito na Tabela 3.

Nos pacientes com isquemia bilateral, foi considerado o membro com o grau mais grave de isquemia. Dos 125 pacientes, $33(26,4 \%)$ tinham sintomas de isquemia do membro inferior direito; $50(40,0 \%)$ tinham isquemia do membro inferior esquerdo e $42(33,6 \%)$ tinham isquemia bilateral dos membros inferiores. Dessa forma, 167 membros isquêmicos e 83 não-isquêmicos com 
Tabela 4 - Achados arteriográficos e ultrassonográficos do conjunto dos segmentos aorto-ilíacos $(\mathrm{n}=552)$

\begin{tabular}{lccc}
\hline & \multicolumn{2}{c}{ Exames } & \\
\cline { 2 - 3 } Grau de estenose & Arteriografia (n) & Eco-Doppler (n) & Concordantes (mesmo grau) \\
\hline $1(0-19 \%)$ & 303 & 294 & 285 \\
$2(20-49 \%)$ & 91 & 85 & 82 \\
$3(50-79 \%)$ & 18 & 28 & 16 \\
$4(80-99 \%)$ & 44 & 45 & 38 \\
$5(100 \%)$ & 96 & 100 & 87 \\
Total & 552 & 552 & 506 \\
\hline
\end{tabular}

algum grau de estenose no território aorto-ilíaco entraram no estudo.

No total de 125 pacientes, 625 segmentos aortoilíacos eram potencialmente comparáveis. Ao longo do estudo, 73 segmentos foram eliminados da análise, por apresentarem um dos critérios de exclusão descritos na seção anterior:

- Seis pacientes (12 segmentos) tinham amputação prévia do membro inferior contralateral;

- Em 24 pacientes (48 segmentos) com isquemia unilateral, com o membro inferior contralateral normal;

- Em 13 segmentos ilíacos, o laudo do exame ultrassonográfico foi inconclusivo, por razões técnicas.

No total, foram avaliados comparativamente $552 \mathrm{seg}$ mentos arteriais. Estenoses hemodinamicamente significativas foram detectadas na AC em 62 segmentos $(11,2 \%)$ e na eco-Doppler em 63 segmentos $(11,4 \%)$. Oclusões segmentares foram observadas nas AC em 96 segmentos $(17,4 \%)$ e nas eco-Doppler, em 100 segmentos $(18,1 \%)$. Portanto, lesões clinicamente relevantes foram observadas nas AC em 158 segmentos $(28,6 \%)$ e nas eco-Doppler em 163 segmentos (29,5\%).

Os achados arteriográficos e ultrassonográficos dos diversos segmentos estudados estão compilados na Tabela 4. A última coluna da tabela mostra o número de exames no qual houve concordância entre os resultados da AC e da eco-Doppler (mesmo grau de estenose).

A Tabela 5 resume a análise estatística dos resultados. Foram encontrados elevados índices de validade em todos os segmentos arteriais examinados. Os testes kappa mostraram ótimos coeficientes de correlação entre os dois exames, em todos os segmentos. Na Tabela 6 , está resumida a análise da validade da eco-Doppler em distinguir estenoses críticas de oclusões.

\section{Discussão}

O diagnóstico inicial de pacientes com DOAI se baseia no exame clínico. Os exames complementares têm a função de confirmar o diagnóstico clínico, localizar o processo patológico e avaliar suas alterações fisiopatológicas. Portanto, os exames complementares são exames de avaliação que permitem planejar o tratamento do processo patológico ${ }^{1,7,11,12}$. Tradicionalmente, a AC tem sido utilizada como o padrão-ouro, isto é, o exame que melhor avalia a doença oclusiva e permite tomar decisões terapêuticas ${ }^{1,7,10-12}$. Mas a arteriografia apresenta limitações importantes como o exame padrãoouro na DOAI. Nessa discussão, o termo limitações não se refere às desvantagens do método (ser invasivo, desconfortável, caro e com risco de complicações graves), mas sim à incapacidade de a AC mostrar a anatomia arterial e as alterações hemodinâmicas causadas pela DOAI. As limitações da AC se devem a vários fatores:

- Distribuição do contraste na luz arterial;

- Espasmo provocado pelo cateter ou pela própria injeção de contraste;

- Dificuldade de se mostrar placas de ateroma nos óstios arteriais;

- Dificuldades de se interpretar o grau de estenose, mesmo em AC de boa qualidade; 
Tabela 5 - Índices de validade para distinguir lesão hemodinamicamente significativa de lesão não significativa

\begin{tabular}{|c|c|c|c|c|c|c|}
\hline \multirow{2}{*}{$\begin{array}{l}\text { Segmento } \\
\text { arterial }\end{array}$} & \multicolumn{6}{|c|}{ Índice de validade (\%) } \\
\hline & Sensibilidade & Especificidade & VPP & VPN & Acurácia & kappa \\
\hline Aorta & 92,8 & 92,6 & 61,9 & 99,0 & 92,7 & 0,82 \\
\hline $\begin{array}{l}\text { Ilíaca comum } \\
\text { direita }\end{array}$ & 90,6 & 94,5 & 87,9 & 95,8 & 93,3 & 0,84 \\
\hline $\begin{array}{l}\text { Ilíaca comum } \\
\text { esquerda }\end{array}$ & 84,4 & 96,2 & 90,0 & 93,7 & 92,7 & 0,82 \\
\hline $\begin{array}{l}\text { Ilíaca externa } \\
\text { direita }\end{array}$ & 90,0 & 93,9 & 90,0 & 93,9 & 92,4 & 0,84 \\
\hline $\begin{array}{l}\text { Ilíaca externa } \\
\text { esquerda }\end{array}$ & 90,0 & 86,2 & 81,8 & 93,4 & 88,9 & 0,81 \\
\hline Total & 89,2 & 93,1 & 83,9 & 95,6 & 92,0 & 0,81 \\
\hline
\end{tabular}

$\mathrm{VPN}=$ valor preditivo negativo; $\mathrm{VPP}=$ valor preditivo positivo

Tabela 6 - Resultados dos exames de estenoses críticas versus oclusões no conjunto dos segmentos aorto-ilíacos $(\mathrm{n}=145)$

\begin{tabular}{lccc}
\hline Tipo de lesão & Arteriografia (n) & Eco-Doppler (n) & Exames correlacionados \\
\hline Estenose crítica & 44 & 45 & 38 \\
Oclusão & 96 & 100 & 87 \\
\hline
\end{tabular}

Índices de validade da eco-Doppler: acurácia total $=86 \%$; especificidade $=86,3 \%$; sensibilidade $=91 \%$; teste kappa $=0,71$; valor preditivo negativo $=90,8 \%$; valor preditivo positivo $=93,5 \%$.

- E principalmente pelo fato de a AC ser um exame morfológico, que não fornece informações fisiológi$\operatorname{cas}^{7,13}$.

A tomada de decisões clínicas na DAOP se baseia no significado hemodinâmico das lesões encontradas nos exames de imagem ${ }^{1,7,10-13}$. Uma estenose pode não alterar a pressão de perfusão e o fluxo distal em condições de repouso. Porém, em condições que requerem aumento do fluxo (como o exercício), a estenose se torna hemodinamicamente significativa, provocando redução da perfusão distalmente à lesão. Estudos experimentais e clínicos estabeleceram que redução de 50\% no diâmetro da artéria (o que corresponde a cerca de $75 \%$ da seção transversa da luz arterial) é o limite a partir do qual uma lesão se torna hemodinamicamente significativa ${ }^{14-18}$. Nos dois extremos (uma artéria ilíaca normal ou totalmente ocluída), as imagens arteriográficas são suficientes para a tomada de decisões clínicas. Entretanto, é comum a AC mostrar lesões ateroscleróticas que provocam redução entre 30 e 70\% do diâmetro da artéria, ou mostrar duas ou mais estenoses em série, no mesmo segmento arterial. As dificuldades em se avaliar o grau de estenose pela $\mathrm{AC}$ levaram alguns investigadores a usar a MAD como um método de se avaliar a importância hemodinâmica das lesões ateroscleróticas aorto-ilíacas ${ }^{15,19-21}$. A MAD é considerada atualmente o método mais acurado para avaliar o significado hemodinâmico de uma estenose do segmento aorto-ilíaco ${ }^{19-23}$. No entanto, tem sido pouco usada na prática, devido às dificuldades técnicas para sua execução ${ }^{24}$.

O estabelecimento do método padrão-ouro deve ser o primeiro passo na execução de estudos comparativos. No presente estudo, todas as arteriografias foram realizadas em pelo menos dois planos (ântero-posterior e oblíquo). Os achados angiográficos foram quantificados através da medida acurada das estenoses, nos clichês radiográficos. As dúvidas em relação a algumas 
lesões foram esclarecidas ou com novas injeções de contraste no segmento suspeito ou com a MAD através do cateter angiográfico. A observância estrita dos protocolos permitiu que a AC (com auxílio eventual da MAD em 19 segmentos) fosse considerada o método padrãoouro para esse estudo.

Diversos estudos com metodologias ligeiramente diferentes compararam eco-Doppler com AC, mostrando altos índices de sensibilidade e de especificidade (80 a 95\%) para a eco-Doppler no segmento aorto-ilíaco $^{8,25-37}$. No presente trabalho, a acurácia para se distinguir uma lesão hemodinamicamente significativa de outra não-significativa foi de $92 \%$. Portanto, a eco-Doppler se mostrou altamente acurada tanto para confirmar a presença de DOAI (sensibilidade elevada) quanto para excluir DOAI nos casos onde o grau de suspeita era baixo (especificidade elevada). Tais resultados estão em concordância com a literatura ${ }^{8,25-37}$.

Os índices elevados de acurácia têm duas implicações: uma é que a eco-Doppler permite identificar com segurança os pacientes com lesões leves a moderadas do segmento aorto-ilíaco, que não provocam sintomas isquêmicos nem redução no influxo para uma reconstrução arterial distal ${ }^{38}$. A outra é que a eco-Doppler permite selecionar os pacientes com lesões mais graves, mas bem localizadas, que são candidatos a terapia intervencionista por cateter ${ }^{39-52}$. Tradicionalmente, os candidatos a essa forma de terapia têm que ser submetidos a dois exames arteriográficos: um exame diagnóstico e um segundo exame, durante o qual é executada a intervenção. Quando uma estenose ou oclusão segmentar curta na artéria ilíaca é identificada pela eco-Doppler, pode-se indicar diretamente o tratamento intervencionista percutâneo, conforme recomendado pelo TASC II ${ }^{11}$.

A crítica mais frequente feita à eco-Doppler é que se trata de um exame diagnóstico cujos resultados dependem do examinador. Na eco-Doppler, diferentemente de outros métodos de imagem, o examinador é o único que "vê" todas as imagens obtidas durante o exame e escolhe aquelas que vão fazer parte do laudo. Nos EUA e na Europa, técnicos (chamados vascular technologists) executam os exames, colhendo as imagens e dados fisiológicos que consideram importantes, para subsequente interpretação por um médico, que assina o laudo. Dessa forma, o exame depende da habilidade e experiência de um técnico, que não é o único responsável pelo laudo emitido pelo laboratório. No Brasil, os exames ultrassonográficos são de responsabilidade de médicos especialistas, que além de executarem o exame, o interpretam e assinam o laudo ${ }^{5,6}$. Tal forma de fazer ultrassonografia vascular torna o exame feito no nosso Brasil mais confiável que os exames executados em outros países. Mas não muda o fato de que a experiência e dedicação do examinador são tão importantes quanto à qualidade do aparelho de ultrassonografia.

$\mathrm{O}$ argumento de que a eco-Doppler depende excessivamente do examinador pode ser rebatido pelo contraargumento de que a AC também depende do examinador. Uma arteriografia de qualidade, por exemplo, depende do posicionamento correto do paciente na mesa de exame, da colocação precisa do cateter na luz da artéria, da injeção de contraste em volume e de concentração adequados e da obtenção das imagens no tempo certo depois da injeção. Todas essas etapas dependem da habilidade e experiência do examinador ${ }^{13,41}$. Portanto, o fato de a eco-Doppler ser examinador-dependente não pode ser um argumento contra tal exame. O que há de novo no caso da ecoDoppler é que o especialista tem que tomar decisões clínicas com base num exame que não sabe interpretar e tem que confiar de forma absoluta no laudo fornecido por outro médico. Tal situação nova perturba muitos cirurgiões vasculares, que acabam por solicitar outros exames com os quais estão mais familiarizados, para confirmar os achados da eco-Doppler.

\section{Conclusão}

No presente estudo, a eco-Doppler apresentou elevados índices de validade e de correlação com a arteriografia na avaliação de pacientes com suspeita de DOAI. Essa conclusão está de acordo com quase todos os trabalhos da literatura ${ }^{8,25-37,39-54}$. A eco-Doppler pode ser usada na investigação primária da DOAI, evitando-se a angiografia pré-intervenção por qualquer método (arteriografia com cateter, tomografia computadorizada e ressonância magnética) na imensa maioria dos pacientes com suspeita de DOAI. 


\section{Agradecimentos}

Aos doutores Enrique Vidal, Aguinaldo de Oliveira e Graciliano José França pela excelência dos exames de eco-Doppler que formam este trabalho e ao Dr. Jeferson Freitas Toregiani, pelo inestimável auxílio no trabalho estatístico.

\section{Referências}

1. Brewster DC. Direct reconstruction for aortoiliac occlusive disease. In: Rutherford RB. Vascular surgery. 5th ed. Philadelphia: WB Saunders; 2000. p. 943-72.

2. Moreira RC. Tratamento cirúrgico da doença oclusiva aorto-ilíaca sem arteriografia pré-operatória. J Vasc Bras. 2002; $1: 47-54$

3. Johnston KW. Role of Doppler ultrasonography in determining the hemodynamic significance of aortoiliac disease. Can J Surg. 1978;21:319-20, 325.

4. Strandness DE Jr. Echo-doppler (duplex) ultrasonic scanning. J Vasc Surg. 1985;2:341-4.

5. Giannnini M. Mapeamento duplex das artérias dos membros inferiores. In: Maffei FH. Doenças vasculares periféricas. $3^{\mathrm{a}}$ ed. Rio de Janeiro: Medsi; 2002. p. 341-6.

6. Coelho NA, Morais Filho D. Ecografia vascular arterial dos membros inferiores e superiores. In: Brito CJ. Cirurgia vascular, cirurgia endovascular e angiologia. $2^{\mathrm{a}}$ ed. Rio de Janeiro: Revinter; 2007. p 241-55.

7. Barnes RW. Evaluating aortoiliac disease: an overview. Perspec Vasc Surg. 1995;9:1-14.

8. Alexander JQ, Leos SM, Katz SG. Is duplex ultrasonography an effective single modality for the preoperative evaluation of peripheral vascular disease? Am Surg. 2002;68:1107-10.

9. Porter JM. Overview. In: Porter JM, editor. Year Book of Vascular Surgery 2000. St. Louis: Year Book Medical Publishers; 2000. p. 1-3.

10. Rutherford RB, Baker JD, Ernst C, et al. Recommended standards for reports dealing with lower extremity ischemia: revised version. J Vasc Surg. 1997;26:517-38.

11. Norgren L, Hiatt WR, Dormandy JA, et al. Inter-society consensus for the management of peripheral arterial disease. Int Angiol. 2007;2:81-157.

12. Hirsch S, Haskal JZ, Herzer NR, et al. ACC/AHA 2005 Practice Guidelines for the management of patients with peripheral arterial disease (lower extremity, renal, mesenteric, and abdominal aortic): a collaborative report from the American Association for Vascular Surgery/Society for Vascular Surgery, Society for Cardiovascular Angiography and Interventions, Society for Vascular Medicine and Biology, Society of Interventional Radiology, and the ACC/AHA Task Force on Practice Guidelines (Writing Committee to Develop Guidelines for the Management of Patients With Peripheral Arterial Disease): endorsed by the American Association of Cardiovascular and Pulmonary Rehabilitation; National Heart, Lung, and Blood Institute; Society for Vascular Nursing; TransAtlantic Inter-Society Consensus; and Vascular Disease Foundation. Circulation. 2006;113:e463-654.
13. Johnston KW, Demorais D, Colapinto RF. Difficulty in assessing the severity of aorto-iliac disease by clinical and arteriographic methods. Angiology. 1981;32:609-14.

14. Baker AR, Prytherch DR, Evans DH, Bell PR. Characterisation of aorto-Iliac arterial stenoses in terms of pressure and flow. Cardiovasc Res. 1985;19:559-66.

15. Baker JD. Hemodynamic assessment of aortoiliac segment. Surg Clin North Am. 1990;70:31-40.

16. May AG, DeWeese JA, Rob CG. Hemodynamic effects of arterial stenosis. Surgery. 1963;53:513-24.

17. Strandness DE, Sumner DS. The effect of geometry on arterial blood flow. In: Strandness DE, Sumner DS. Hemodynamics for Surgeons. New York: Grune \& Stratton; 1975. p. 96-119.

18. Sumner DS. Essential hemodynamic principles. In: Rutherford RB. Vascular Surgery. 5th ed. Philadelphia: WB Saunders; 2000. p. 73-120.

19. Moore WS, Hall AD. Unrecognized aortoiliac stenosis: a physiologic approach to the diagnosis. Arch Surg. 1971;103:633-8.

20. Brener BJ, Raines JK, Darling RC, Austen WG. Measurement of systolic femoral artery pressure during reactive hyperemia: an estimate of aortoiliac disease. Circulation. 1974;50:259-67.

21. Udoff EJ, Barth KH, Harrington DP, Kaufman SL, White RI. Hemodynamic significance of iliac artery stenosis: pressure measurements during angiography. Radiology. 1979;132:289-93.

22. Breslau PJ, Jorning PJG, Greep JM. Assessment of aortoiliac disease using hemodynamic measures. Arch Surg. 1985; 120:1050-2.

23. Strauss AL, Roth FJ, Rieger H. Noninvasive assessment of pressure gradients across iliac artery stenoses: duplex and catheter correlative study. J Ultrasound Med. 1993;12:17-22.

24. McWilliams RG, Robertson I, Smye SW, Wijesinghe L, Kessel D. Sources of error in intra-arterial pressure across a stenosis. Eur J Vasc Endovasc Surg. 1998;15:535-40.

25. Legemate DA, Teeuwen C, Hoeneveld H, Ackerstaff RGA, Eikelboom BC. The potential of duplex scanning to replace aorto-iliac and femoro-popliteal angiography. Eur J Vasc Surg. 1989;3:49-54

26. Cossman DV, Ellison JE, Wagner WH, et al. Comparison of contrast arteriography to arterial mapping with color-flow duplex imaging in the lower extremities. J Vasc Surg. 1989;10:522-8

27. Legemate DA, Teeuwen C, Hoeneveld H, Eikelboom BC. Value of duplex scanning compared with angiography and pressure measurement in the assessment of aortoiliac arterial lesions. Br J Surg. 1991;78:1003-8.

28. Rosfors S, Eriksson M, Hoglund N, Johansson G. Duplex ultrasound in patients with suspected aorto-iliac occlusive disease. Eur J Vasc Surg.1993;7:513-7.

29. de Smet AA, Visser K, Kitslaar PJ. Duplex scanning for grading aortoiliac obstructive disease and guiding treatment. Eur J Vasc Surg. 1994;8:711-5.

30. AbuRahma AF, Khan S, Robinson PA. Selective use of segmental Doppler pressures and color duplex imaging in the localization of arterial occlusive disease of the lower extremity. Surgery. 1995;118:496-503. 
31. de Smet AA, Ermers EJ, Kitslaar PJ. Duplex velocity characteristics of aortoiliac stenoses. J Vasc Surg. 1996;23:628-36.

32. Krnic A, Vucic N, Sucic Z. Duplex scanning compared with intra-arterial angiography in diagnosing peripheral arterial disease: three analytical approaches. Vasa. 2006;35:86-91.

33. Favaretto E, Pili C, Amato A, et al. Analysis of agreement between Duplex ultrasound scanning and arteriography in patients with lower limb artery disease. J Cardiovasc Med (Hagerstown). 2007;8:337-41.

34. Allard L, Cloutier G, Durand LG, Roederer GO, Langlois YE. Limitations of ultrasonic duplex scanning for diagnosing lower limb arterial stenosis in the presence of adjacent segment disease. J Vasc Surg. 1994;19:650-7.

35. Bergamini TM, Tatum CM Jr, Marshall C, Hall-Disselkamp B, Richardson JD. Effect of multilevel sequential stenosis on lower extremity arterial duplex scanning. Am J Surg. 1995;169:564-6.

36. Sensier Y, Hartshorne T, Thrush A, Handford H, Nydahl S, London NJ. The effect of adjacent segment disease on the accuracy of duplex scanning for the diagnosis of lower limb arterial disease. Eur J Vasc Endovasc Surg. 1996;12:238-42.

37. Aly S, Jenkins MP, Zaidi FH, Coleridge Smith PD, Bishop CC. Duplex scanning and effect of multisegmental arterial disease on its accuracy lower limb arteries. Eur J Vasc Endovasc Surg. 1998;16:345-9.

38. Jacobovicz, C, Timi JR, França LH, Stahlke Jr. HJ, Nakahara J. Avaliação do eco-Doppler na predição da necessidade de arteriografia do território aorto-ilíaco em pacientes submetidos a revascularização arterial infra-inguinal. J Vasc Bras. 2004;3:5-12.

39. Back MR, Bowser AN, Schmacht DC, Johnson BL, Bandyk DF. Duplex selection facilitates single point-of-service endovascular and surgical management of aortoiliac occlusive disease. Ann Vasc Surg. 2002;16:566-74.

40. Karkowski J, Zarins CK. Endografting of the abdominal aorta and iliac arteries for occlusive disease. J Cardiovasc Surg (Torino). 2005;46:349-57.

41. Queral LA, Lucas PR, Badder EM, Wilkerson RJ. Lower extremity revascularization based on intraoperative arteriography. Ann Vasc Surg. 2007;21:284-8.

42. Vashisht R, Ellis MR, Skidmore C, Blair SD, Greenhalgh RM, O'Malley MK. Colour-coded duplex ultrasonography in the selection of patients for endo-vascular surgery. Br J Surg. 1992;79:1030-1.

43. van der Heijden FH, Legemate DA, van Leeuwen MS, Mali WP, Eikelboom BC. Value of duplex scanning in the selection of patients for percutaneous transluminal angioplasty. Eur J Vasc Surg. 1993;7:71-6.
44. Elsman BH, Legemate DA, van der Heijden FH, de Vos HJ, Mali WP, Eikelboom BC. Impact of ultrasonografhic duplex scanning on therapeutic decision making in lower-limb arterial disease. Br J Surg. 1995;82:630-3.

45. Pemberton M, Nydahl S, Hartshorne T, Naylor AR, Bell PR, London NJ. Colour-coded duplex imaging can safely replace diagnostic arteriography in patients with lower-limb arterial disease. Br J Surg. 1996;83:1725-8.

46. Schneider PA, Ogawa DY. Is routine preoperative aortoiliac arteriography necessary in the treatment of lower extremity ischemia? J Vasc Surg.1998;28:28-34.

47. van der Zaag ES, Legemate DA, Nguyen T, Balm R, Jacobs MJ. Aortoiliac reconstructive surgery based upon the results of duplex scanning. Eur J Vasc Endovasc Surg. 1998;16:383-9.

48. Katsamouris AN, Giannoukas AD, Tsetis D, Kostas T, Petinarakis I, Gourtsoyiannis N. Can ultrasound replace arteriography in the management of chronic arterial occlusive disease of the lower limb? Eur J Vasc Endovasc Surg. 2001;21:155-9.

49. Alexander JQ, Leos SM, Katz SG. Is duplex ultrasonography an effective single modality for the preoperative evaluation of peripheral vascular disease? Am Surg. 2002;68:1107-10.

50. Boström A, Karacagil S, Löfberg AM, et al. Selection of patients with lower limb arterial occlusive disease for endovascular treatment of the iliac arteries with duplex scanning. Vasc Surg. 2001;35:437-42.

51. Cardia G, Cianci V, Iusco D, Nacchiero M. Ultrasound duplex as a sole exam for surgical purposes in lower limb arterial obstructive disease. Minerva Cardioangiol. 2001;49:349-55.

52. Luján S, Criado E, Puras E, Izquierdo LM. Duplex scanning or arteriography for preoperative planning of lower limb revascularization. Eur J Vasc Surg. 2002;24:31-6.

53. Leiner T, Tordoir JH, Kessels AG, et al. Comparison of treatment plans for peripheral arterial disease made with multistation contrast medium-enhanced magnetic resonance angiography and duplex ultrasound scanning. J Vasc Surg. 2003;37:1255-62.

54. Collins R, Burch J, Cranny G, et al.Duplex ultrasonography, magnetic resonance angiography and computed tomography angiography for diagnosis and assessment of symptomatic, lower limb peripheral arterial disease: systematic review. BMJ. 2007;334:1257-69.

Correspondência:

Ricardo Rocha Moreira

Rua Pedro Muraro 50, casa 24

CEP 82030-620 - Curitiba, PR

Tel.: (41) 3335.3233, 3244.8787

E-mail: ina@onda.com.br 
Anexo 1

Critérios para quantificação de estenoses

Critérios para quantificação de estenoses

Modo B (preto e branco convencional) ou modo colorido (incluindo angio): imagem do vaso examinado em sentido transversal. Calculado o percentual de estenose, seja por área (melhor) ou por diâmetro, com possibilidade de medir a luz residual.

Modo Doppler ou análise da onda de fluxo (em caso de discordâncias entre estes vários critérios, este é o critério que terá prioridade para determinar o grau de estenose):

- Análise de velocidades absolutas (sistólica, diastólica e média) e a obtenção de índices: resistência (sistólica-diastólica divididas pela sistólica) e pulsatilidade (sistólica-diastólica divididas pela média). É importante também a determinação do tempo de aceleração: tempo transcorrido entre o começo da onda sistólica e o pico ou velocidade máxima.

Resumo dos critérios do registro Doppler

Grau de estenose (fluxo) Janela sistólica

Estenose $<20 \%$

Trifásico alargamento espectral Normal

Estenose $20-50 \%$

Trifásico alargamento espectral

Trifásico ausente

Aumento < 100\%

Estenose $50-79 \%$

Mono ou bifásico ausente

Aumento 100-200\%

Estenose 80-99\%

Monofásico ausente

Aumento $>200 \%$

Oclusão

Ausente presente

Diminuída

* VPS: velocidade de pico sistólico.

Critérios acessórios:

- Tempo de aceleração na femoral comum: quando $>140 \mathrm{~m} / \mathrm{s}$ infere estenose significativa proximal, com acurácia total de aproximadamente $94 \%$;

- Velocidade diastólica: $>100 \mathrm{~cm} / \mathrm{s}$ no local: indica estenose severa superior a 75\%;

- Aumento do fluxo diastólico femoral registrado após manobra de compressão femoral (hiperemia reativa): infere estenose significativa proximal (> 50\%);

- Índice de pulsatilidade: segmento aorto-ilíaco: entre 2-6; femoral comum: entre 4-13. 